\title{
Esfera em Plano Inclinado: Conservação da Energia Mecânica e Força de Atrito
}

(Sphere rolling down in a Rough Inclined Plane: Conservation of the Mechanical Energy and Frictional Force)

\author{
Wilton Pereira da Silva ${ }^{a *}$ Cleide M. D. P. S. e Silva ${ }^{b}$, Jürgen W. Precker $^{c}$ \\ Diogo D. P. S. e Silva ${ }^{\dagger}$, Ivomar Brito Soares ${ }^{\dagger}$ e Cleiton D. P. S. e Silva ${ }^{\ddagger}$ \\ ${ }^{a b c}$ Grupo de Aquisição e Tratamento de Dados, Departamento de Física, \\ Universidade Federal de Campina Grande, 58109-970, PB, Brasil
}

Recebido em 11 de julho, 2003. Aceito em 31 de outubro, 2003.

\begin{abstract}
Este artigo propõe um experimento visando demonstrar que a força de atrito estático é conservativa. O experimento consiste em uma esfera rolando em um plano inclinado, para o qual a inclinação pode ser variada. Para cada ângulo estabelecido a energia mecânica no início e no fim da pista foi determinada. A análise dos resultados mostrou que a conservação da energia mecânica ocorre até um ângulo limite, a partir do qual há escorregamento. A determinação deste ângulo por inspeção gráfica possibilitou o cálculo do coeficiente de atrito estático entre a pista e a esfera em movimento.
\end{abstract}

\begin{abstract}
This paper proposes an experiment seeking to demonstrate that for the static frictional force there is conservation of energy. The experiment consists of a sphere rolling down in a rough inclined plane, for which the slope can be varied. For each given angle the mechanical energy in the beginning and at the end of the movement was determined. The analysis of the results showed that the conservation of the mechanical energy occurs up to a maximum angle, starting from the one which the sphere begins to slide. The determination of this angle by graphic inspection allowed the calculation of the coefficient of static friction between the bodies.
\end{abstract}

\section{Introdução}

A vivência em sala de aula possibilita constatar que uma parcela considerável de alunos fazem uma certa confusão ao relacionar os tópicos "Conservação de Energia" e "Força de Atrito". Tais alunos acreditam que a força de atrito, tanto cinético quanto estático, indistintamente, seja uma força não-conservativa. Uma interessante discussão sobre o assunto "Força de Atrito" é apresentada em [1] e em [2]. Por mais que livros didáticos [3] e artigos em revistas especializadas (ver [4-6], por exemplo) abordem o assunto "Força de Atrito", de uma forma geral, a confusão sobre os tópicos mencionados permanece presente na visão de muitos alunos. Uma pesquisa bibliográfica minuciosa mostra que o assunto, embora seja estudado há tempos, é bastante atual (ver, por exemplo, [7-12]). Apesar da ampla e recente bibliografia disponível sobre atrito, um problema permanece pouco explorado: a confusão, por parte de alguns alunos, a respeito dos temas "força de atrito" e "conservação de energia".

Para tentar minimizar o problema, pelo menos em sua área de atuação, os dois primeiros autores deste artigo desenvolveram um experimento simples. Tal experimento consiste em uma esfera abandonada a partir do repouso, sobre

\footnotetext{
*wiltonps@uol.com.br

${ }^{\dagger}$ Bolsistas do projeto "Aperfeiçoamento de Professores no Ensino de Física"

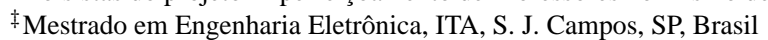

um plano inclinado, rolando pista abaixo. O propósito do experimento se adequa à idéia de "ver para crer": a energia mecânica da esfera deve ser determinada no início e no fim da pista, para várias inclinações diferentes. Então, através da análise dos dados obtidos, pode-se tirar uma conclusão sobre a relação entre a energia mecânica inicial e final da esfera. Tal conclusão possibilita obter uma resposta imediata sobre o questionamento com relação à força de atrito estático e à conservação de energia mecânica.

\section{Montagem Experimental e Medi- das Realizadas}

Para realizar o experimento usou-se o KEM [13], que é um "Kit para Experiências de Mecânica" disponível em várias escolas de nível médio e em algumas universidades. O KEM foi fixado nas bordas de uma bancada, conforme mostram as Figs. 1 e 2.

No experimento foi usada uma esfera de aço (massa $m$ $=0.04490 \mathrm{~kg}$ e raio $R=0,01110 \mathrm{~m}$ ) sobre a pista do KEM, de comprimento $x=0,400 \mathrm{~m}$. O ângulo $\theta$ entre a direção do 


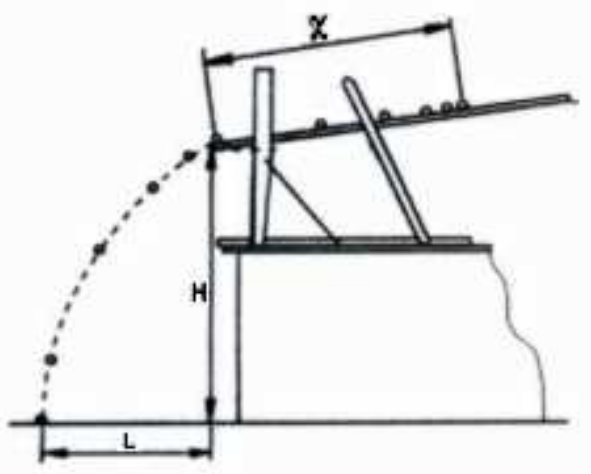

Figura 1. Perfil da montagem experimental: KEM sobre a bancada.

movimento e a horizontal estabelecido com auxílio de um transferidor acoplado ao plano que contém a pista e de um prumo. Para "zerar" o transferidor, houve uma compensação para a resistência ao rolamento [14]: a inclinação zero da pista foi tal que o movimento da esfera, ao sofrer um pequeno impulso, pudesse ser considerado uniforme (se a pista fosse muito longa, a esfera continuaria em movimento retilíneo e uniforme em todo o trajeto). Estabelecido este "ângulo zero", a pista foi inclinada de 4,0 em $4,0^{\circ}$, a partir de $14,0^{\circ}$, até $54,0^{\circ}$. Uma observação: para ângulos muito pequenos, haveria a possibilidade da componente do peso na direção da pista (pequena, nesses casos) ser "parcialmente mascarada" pela resistência ao rolamento, muito embora, conforme mencionado, cuidados tivessem sido tomados para compensar tais forças de resistência. Por isso, o ângulo inicial foi feito igual a $14^{0}$. Para cada ângulo a esfera foi abandonada em repouso, no ponto superior da pista, e descia plano abaixo. Então, ao sair da pista a uma altura $H$ (ver Figs. 1 e 2), a esfera descrevia uma trajetória parabólica e colidia com o chão, onde havia uma folha de papel ofício,

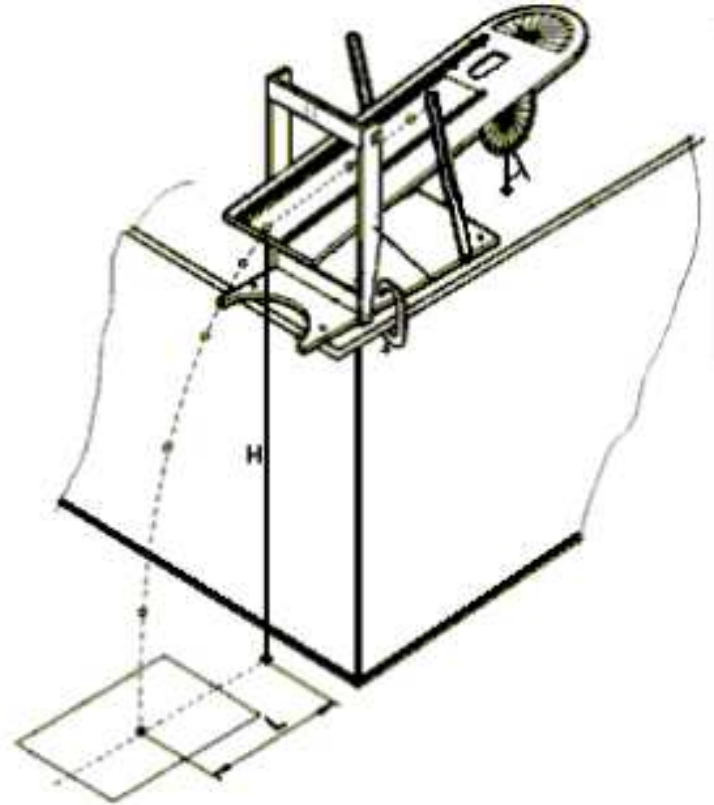

Figura 2. Corpo básico do KEM fixado nas bordas da bancada por um grampo. $\mathrm{O}$ ângulo que indica a inclinação do sistema é medido através do transferidor preso ao plano que contém a pista por onde rola a esfera.

sobre a qual foi colocado uma folha de papel carbono. Assim, o alcance $L$ da esfera em movimento parabólico podia ser medido, pois a projeção da saída da pista no chão também tinha sido marcada sobre o papel ofício, com o auxílio de um prumo, para cada ângulo estabelecido.

Os procedimentos anteriormente descritos possibilitaram realizar as medidas de $H$ e $L$ para cada $\theta$, o que gerou a Tab. 1. Com isso, pode-se calcular o que, a priori, se pretendia: a energia mecânica da esfera no início e no fim da pista.

\begin{tabular}{|l|l|l|l|l|l|l|l|l|l|l|l|}
\hline$\theta\left(^{\circ}\right)$ & 14,0 & 18,0 & 22,0 & 26,0 & 30,0 & 34,0 & 38,0 & 42,0 & 46,0 & 50,0 & 54,0 \\
\hline$H(\mathrm{~m})$ & 0,905 & 0,897 & 0,889 & 0,883 & 0,877 & 0,870 & 0,865 & 0,858 & 0,852 & 0,848 & 0,843 \\
\hline$L(\mathrm{~m})$ & 0,460 & 0,485 & 0,502 & 0,513 & 0,510 & 0,505 & 0,494 & 0,464 & 0,438 & 0,401 & 0,370 \\
\hline
\end{tabular}

Tabela 1. Lançamento de projétil: altura $H$ de lançamento e alcance $L$ para cada ângulo estabelecido.

\section{Determinação da Energia Mecâni- ca da Esfera}

Como a esfera foi sempre abandonada em repouso, no início da pista, a sua energia mecânica inicial é a própria energia potencial gravitacional $E_{p}$. Para determinar $E_{p}$, atribuiu-se o nível de energia potencial zero ao ponto central da esfera quando ela passava pelo ponto mais baixo da pista. Dessa forma, a determinação da energia potencial inicial para cada ângulo $\theta$ requer o cálculo da altura $h$ do ponto central da esfera, quando ela é colocada, em repouso, no ponto mais alto:

$$
h=x \operatorname{sen} \theta,
$$

onde $x$ é o comprimento da pista.

Já a energia mecânica no fim da pista é a própria energia cinética do corpo neste ponto. Para calcular essa energia cinética da esfera é necessário conhecer a velocidade de seu centro de massa na saída da pista. Isto pode ser feito facilmente, lembrando que a parte final do movimento é um lançamento de projétil para o qual se pode desprezar a resistência do ar. Tal movimento é a composição de um Movi- 
mento Retilíneo Uniforme na direção horizontal com um Movimento Retilíneo Uniformemente Variado na vertical, e isso resulta em [15]

$$
V_{C M}=\frac{L}{\cos \theta} \sqrt{\frac{g}{2(H-L \cdot \operatorname{tg} \theta)}}
$$

em que pode-se considerar $g=9,81 \mathrm{~m} / \mathrm{s}^{2}$. Com os dados da Tab. 1 e as Eq. (1) e (2) obtêm-se os resultados parciais indicados na Tab. 2.

\begin{tabular}{|l|l|l|l|l|l|l|l|l|l|l|l|}
\hline$\theta\left(^{\circ}\right)$ & 14,0 & 18,0 & 22,0 & 26,0 & 30,0 & 34,0 & 38,0 & 42,0 & 46,0 & 50,0 & 54,0 \\
\hline$V_{C M}(\mathrm{~m} / \mathrm{s})$ & 1,18 & 1,31 & 1,45 & 1,59 & 1,71 & 1,85 & 2,01 & 2,08 & 2,21 & 2,27 & 2,41 \\
\hline$h(\mathrm{~m})$ & 0,0968 & 0,124 & 0,150 & 0,175 & 0,200 & 0,224 & 0,246 & 0,268 & 0,288 & 0,306 & 0,324 \\
\hline
\end{tabular}

Tabela 2. Velocidade $V_{C M}$ do centro de massa na saída da pista e altura $h$ do centro de massa da esfera no início da pista (com relação ao nível zero de energia potencial) para cada ângulo fixado.

Com os dados da Tab. 2 já se pode calcular a energia mecânica inicial da esfera, através da conhecida expressão

$$
E_{m i}=m g h .
$$

Por outro lado, a energia mecânica no final da pista reduz-se à energia cinética da esfera, que é dada por

$$
E_{m f}=(1 / 2) m V_{C M}^{2}+(1 / 2) I_{C M} \omega^{2},
$$

onde $\omega$ é a velocidade angular devido à rotação e $I_{C M}$ (igual a (2/5) $m R^{2}$, sendo $R$ o raio) é o momento de inércia da esfera em relação ao eixo de giro. Supondo que a esfera role sem deslizar, tem-se a relação $V_{C M}=\omega R$ e, então,

$$
E_{m f}=(7 / 10) m V_{C M}^{2} .
$$

Com os dados da Tab. 2 e as Eq (3) e (5) pode-se montar a Tab. 3, que dá a energia mecânica inicial e final para os ângulos estabelecidos.

\begin{tabular}{|l|l|l|l|l|l|l|l|l|l|l|l|}
\hline$\theta\left({ }^{\circ}\right)$ & 14,0 & 18,0 & 22,0 & 26,0 & 30,0 & 34,0 & 38,0 & 42,0 & 46,0 & 50,0 & 54,0 \\
\hline$E_{m i}(\mathbf{J})$ & 0,0426 & 0,0546 & 0,0661 & 0,0771 & 0,0881 & 0,0987 & 0,108 & 0,118 & 0,127 & 0,135 & 0,143 \\
\hline$E_{m f}(\mathbf{J})$ & 0,0438 & 0,0539 & 0,0661 & 0,0795 & 0,0919 & 0,108 & 0,127 & 0,136 & 0,154 & 0,162 & 0,183 \\
\hline
\end{tabular}

Tabela 3. Energia mecânica inicial e final para cada ângulo fixado.

Para interpretar os resultados finais, dados na Tab. 3, os pontos $\left(\mathrm{E}_{m i} ; \mathrm{E}_{m f}\right)$ foram plotados através do software LABFit [16]. O gráfico obtido foi reduzido com o uso do software IrfanView [17]. O resultado é mostrado na Fig. 3.

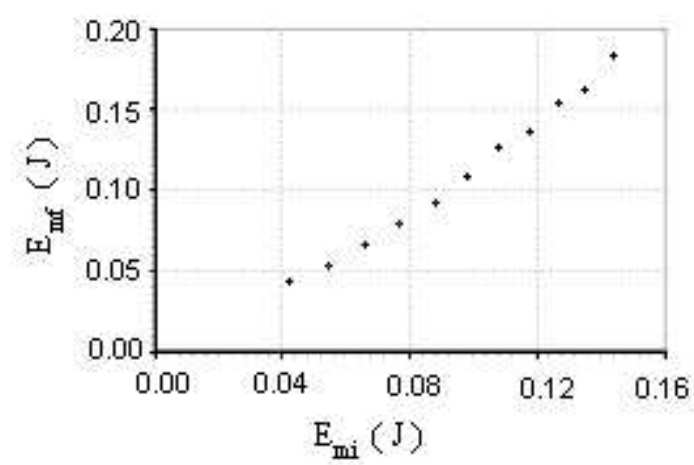

Figura 3. Pontos relativos à Tab. 3: energia mecânica final versus energia mecânica inicial.

Aqui cabe um comentário: o propósito inicial do experimento foi o de "mostrar" que a energia mecânica é conservada durante o rolamento da esfera sobre a pista. Entretanto, uma inspeção visual da Fig. 3 mostra que os pontos não se adequam a uma reta que passa pela origem, com coeficiente angular próximo do valor 1 , condições necessárias para que se tenha a reta $E_{m f}=E_{m i}$, que indicaria a conservação de energia. Essa constatação requer uma análise mais aprofundada da causa do possível insucesso do experimento, e isso será feito a seguir.

\section{Análise dos Dados Obtidos}

Rápidas análises indicam o porquê do aparente fracasso com relação ao objetivo inicial: o experimento se prolongou além dos "ângulos adequados", para os quais haveria conservação de energia. Mas se essa explicação for correta, há que se identificar uma região de pontos em que a suposição inicial $E_{m f}=E_{m i}$ seja verdadeira. Para tanto, sobre os pontos da Fig. 3 foi traçada uma reta impondo-se a condição $E_{m f}=E_{m i}$. O gráfico obtido é mostrado na Fig. 4.

A análise da Fig. 4 mostra claramente que os pontos de 1 a 4 se "encaixam" na reta imposta. O quinto ponto está um pouco fora dessa reta, mas isso poderia ser devido a erros estatísticos e não sistemáticos. Para avaliar essa pos- 
sibilidade, uma reta $E_{m f}=a_{1} E_{m i}+a_{2}$ foi ajustada aos 5 primeiros pontos experimentais, o que gerou a Fig. 5. Essa figura mostra a função ajustada (linha central), a faixa com 95,4\% de confiança para a função ajustada (linhas externas contínuas) e a faixa de predição dos pontos experimentais (linhas tracejadas), também com 95,4\% de confiança. Detalhes sobre a definição dessas faixas podem ser obtidos, por exemplo, em [18] e em [19].

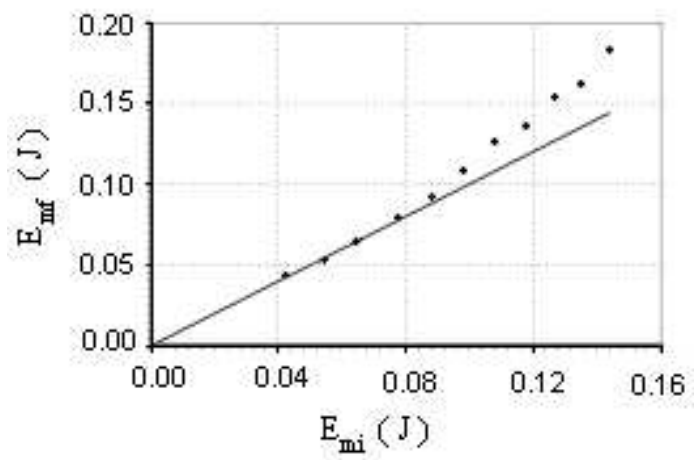

Figura 4. Pontos experimentais e a reta imposta: $E_{m f}=\mathrm{E}_{m i}$.

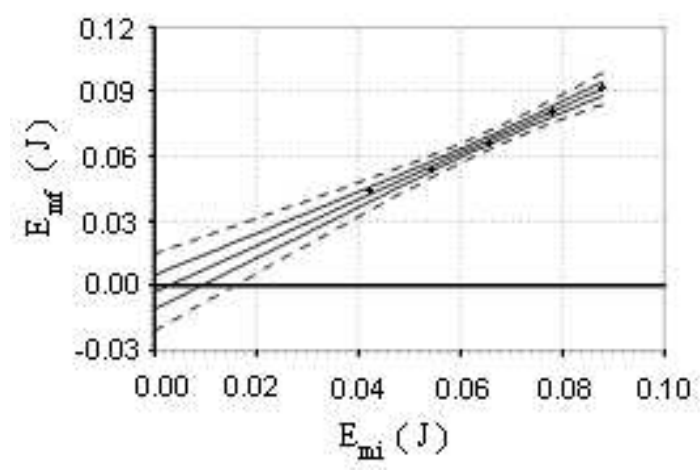

Figura 5. Ajuste com extrapolação até $\mathrm{E}_{m i}=0$. A linha central é a função ajustada. As duas linhas externas contínuas definem a faixa de confiança da função ajustada (com 95,4\% de confiança). As duas linhas tracejadas definem a faixa de predição dos pontos experimentais (com $95,4 \%$ de confiança).

Uma extrapolação da função ajustada para $E_{m i}=0$ aponta para $E_{m f}=(-0,003 \pm 0,009) \mathrm{J}$, com 95,4\% de confiança, o que não possibilita descartar o valor zero para $E_{m f}$. Isto significa que o quinto ponto é aceitável na definição de uma reta passando pela origem. Análises similares supondo a inclusão de outros pontos já apontariam para a presença de erros sistemáticos importantes. Fazendo, por exemplo, o ajuste de uma reta aos 7 primeiros pontos experimentais obtém-se, para $E_{m i}=0: E_{m f}=(-0,014 \pm$ 0,016 ) $\mathbf{J}$, com $95,4 \%$ de confiança. Este resultado (o intervalo contém o zero, mas nas vizinhanças do limite superior) praticamente possibilita descartar o sétimo ponto ao se definir uma reta passando pela origem. Simulações semelhantes, explorando todas as possibilidades, indicam que apenas os 5 primeiros pontos se adequam, com razoável segurança, a uma reta passando pela origem. Então, ajustando-se a função $E_{m f}=a_{1} E_{m i}$ aos 5 primeiros pontos, obtém-se $a_{1}=1,023$ (com desvio de $\sigma_{a 1}=0,010$ ), o que resulta no gráfico mostrado na Fig. 6.

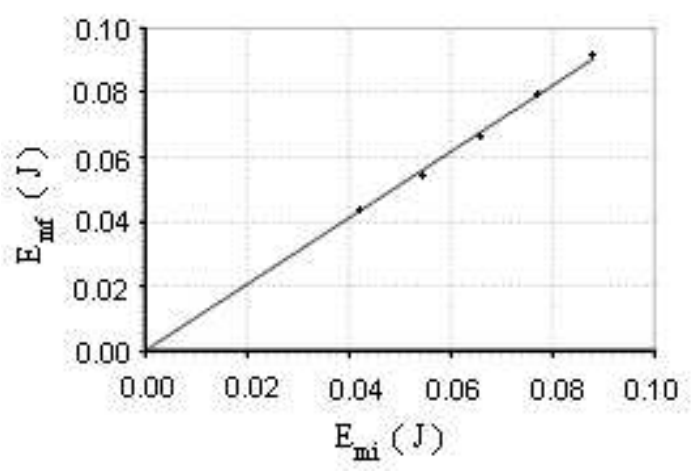

Figura 6. Ajuste da reta passando pela origem aos 5 primeiros pontos experimentais: $\mathrm{E}_{m f}=1,023 \mathrm{E}_{m i}$.

Assim, a afirmação de que para os 5 primeiros pontos a relação $E_{m f}=E_{m i}$ seja verdadeira contém um erro de $2,3 \%$. Por outro lado, traçando a reta $E_{m f}=1,023 E_{m i}$ em um gráfico com todos os pontos experimentais, obtém-se o resultado mostrado na Fig. 7.

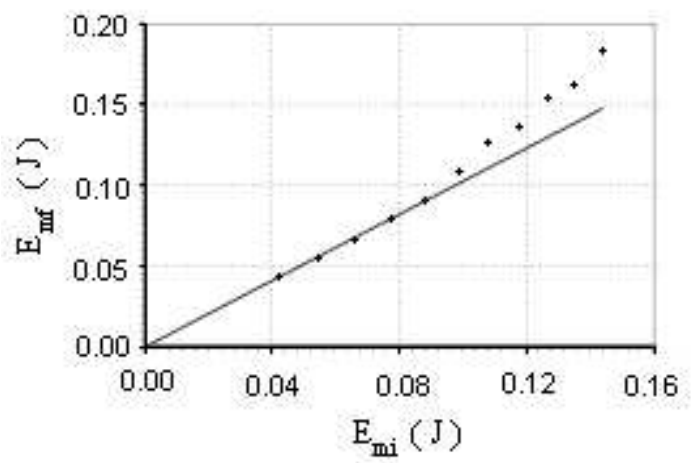

Figura 7. Todos os pontos experimentais e a reta $E_{m f}=$ $1,023 E_{m i}$ : para a região formada pelos 5 primeiros pontos há a conservação de energia.

Para os pontos fora desta primeira região formada pelos 5 primeiros pontos, pode-se descartar a conservação de energia e isso significa que a esfera já desliza sobre a pista.

Uma observação pouco atenta da Fig. 7 poderia levar à suposição de que, a partir do sexto ponto, "a energia mecânica final esteja aumentando mais que a inicial", com o aumento do ângulo. Na verdade, a suposição correta deveria ser a de que a esfera já esteja deslizando. Logo, a relação $V_{C M}=\omega R$ já não é mais verdadeira e, portanto, a energia mecânica no fim da pista não poderia mais ser calculada pela Eq. (5). Isso se deve ao fato de que, como há deslizamento, a velocidade de translação é maior do que seria sem deslizamento, causando uma superestimação da energia de rotação. 


\section{Determinação do Coeficiente de Atrito Estático entre a Esfera e a Pista}

Muito embora não fizesse parte dos objetivos originais do experimento, pode-se fazer uma boa estimativa do valor do coeficiente de atrito estático entre as superfícies envolvidas, por inspeção gráfica. Isso é possível devido ao "acidente" experimental: a inclinação da pista aumentou tanto, que a esfera começou a deslizar. Nesse caso, a energia mecânica já não era conservada durante o movimento. Mas para que seja determinado o coeficiente de atrito estático, há que se estudar um pouco mais. Uma revisão semelhante à que aqui será desenvolvida para uma esfera é apresentada, por exemplo, em [20], para um cilindro.

\subsection{Aplicação das Leis de Newton a uma Es- fera que Rola sem Deslizar}

A Fig. 8 (a) mostra um diagrama esquemático de uma esfera de massa $m$ e raio $R$ rolando pista abaixo. Já a Fig. 8 (b) destaca o diagrama de corpo livre para essa esfera.
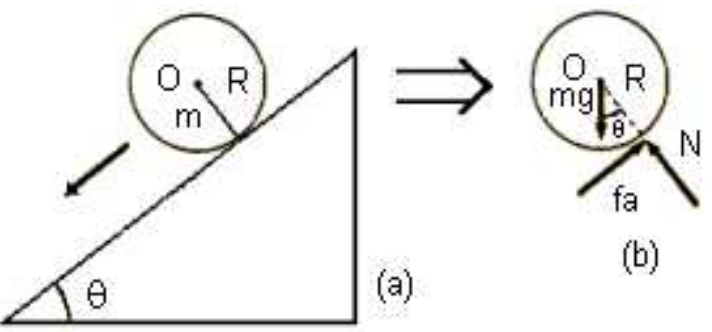

(b)

Figura 8. (a) Esfera rolando num plano inclinado. (b) Diagrama de corpo livre para a esfera: força peso $(\mathrm{mg})$, normal $(\mathrm{N})$ e força de atrito $\left(\mathrm{f}_{a}\right)$.

A expressão da aceleração linear $a$ do centro de massa é obtida aplicando a segunda lei de Newton à translação da esfera:

$$
a=g \operatorname{sen} \theta-\frac{f_{a}}{m}
$$

em que $g$ é a aceleração da gravidade e $f_{a}$ é a força de atrito estático sobre o corpo. Aplicando-se a segunda lei de Newton à rotação da esfera, com a soma dos momentos em relação a um eixo passando pelo seu centro de massa $\mathrm{O}$, tem-se:

$$
f_{a} \cdot R=I_{C M} \alpha,
$$

em que $\alpha$ é a aceleração angular da esfera, dada por $\alpha=$ $a / R$. Combinando as Eq. (6) e (7), obtém-se:

$$
f_{a}=\frac{g \operatorname{sen} \theta}{\frac{R^{2}}{I_{C M}}+\frac{1}{m}} .
$$

Por outro lado, a condição de não escorregamento, e portanto de conservação da energia mecânica, é dada por

$$
f_{a} \leq \mu_{e} N
$$

Lembrando que $N=m g \cos \theta$ e substituindo a Eq. (8) na Eq. (9), obtém-se

$$
\operatorname{tg} \theta \leq\left(\frac{m R^{2}}{I_{C M}}+1\right) \mu_{e} .
$$

Muito embora o experimento envolva uma esfera, a Eq. (10) estabelece a condição para que um corpo role sem deslizar em um plano com inclinação $\theta$, de uma forma geral. Então, a Eq. (10) dá a condição de "conservação de energia mecânica”. Para o caso específico da esfera, pode-se reescrever a Eq. (10) na situação limite, que é a da igualdade, isolando o coeficiente de atrito estático:

$$
\mu_{e}=\frac{2}{7} \operatorname{tg} \theta
$$

\subsection{Estimativa de um Coeficiente de Atrito Estático "Efetivo"}

Fazendo uma nova inspeção na Fig. 7, tendo em conta a Eq. (11), pode-se afirmar, com razoável segurança, mesmo levando em consideração as flutuações estatísticas, que o ângulo para o qual começou o escorregamento da esfera está dentro do intervalo

$$
\theta=(32,0 \pm 2,0)^{0}
$$

referente ao trecho compreendido entre os pontos $5(\theta=$ $\left.30,0^{0}\right)$ e $6\left(\theta=34,0^{\circ}\right)$, com centro em $\theta=32,0^{\circ}$.

Substituindo o valor dado em (12) na Eq. (11) encontrase, por propagação de erros (ver, por exemplo, [19] (Cap. 4) e [21]) o seguinte resultado:

$$
\mu_{e}=(0,179 \pm 0,014)
$$

\section{Discussões e Conclusões}

Um dos resultados esperados para o experimento seria a constatação, por parte de alunos, de que a força de atrito estático é uma força conservativa, diferentemente da força de atrito cinético. Esta constatação se daria pelo ajuste dos pontos experimentais a uma reta passando pela origem, com um valor obtido para o coeficiente angular próximo de 1. Para os 5 primeiros pontos experimentais este objetivo foi alcançado, com erro de $2,3 \%$, o que é bastante razoável. Surgiu, entretanto, um elemento não esperado, que seria a possibilidade da determinação do coeficiente de atrito estático, mesmo com a esfera em movimento, e portanto com o sistema sujeito a perturbações produzidas pelo movimento que levem a variações no valor da componente normal da força de contato entre a esfera e a pista, ao longo do percurso.

Naturalmente, a determinação do coeficiente de atrito estático apresentada neste artigo embute o fato de que o ângulo de escorregamento não foi determinado, mas sim estimado por inspeção gráfica, e isso pode causar um certo 
desconforto. Em contrapartida, esta determinação se deu na condição real de movimento da esfera. Devido às imperfeições da pista (ondulações) e da esfera (excentricidade e "ovalização"), o próprio movimento é perturbado, e isso provoca o efeito de iniciar o escorregamento em ângulos bem menores que aqueles para um sistema ideal. Como se sabe, a perturbação de um sistema "mascara" a força de atrito e este fato, inclusive, é um recurso bastante utilizado para minimizar erros devido ao atrito em muitos experimentos em que tais forças são indesejáveis. Exemplos extremos de minimização da força de atrito podem ser encontrados em experimentos envolvendo um trilho de ar.

Para o sistema isolado, sem perturbação, no qual se aplica uma força de arrasto na esfera em repouso sobre a pista na horizontal, experimentos paralelos ao apresentado aqui apontaram para o valor 0,3 para o coeficiente de atrito estático entre o aço da esfera e a pista. Mas tal valor, no experimento deste artigo, indicaria a situação de "não escorregamento", isto é, de conservação de energia mecânica até uma inclinação da pista aproximadamente igual a $46^{\circ}$. Obviamente, conforme foi visto, o escorregamento aconteceu muito antes, e essa "surpresa" foi um outro fato que motivou este artigo.

Discussões sobre força de atrito estático associada a perturbações mecânicas normalmente não fazem parte do conteúdo de livros-texto destinados a cursos básicos de Física (nível superior). Um experimento deste tipo - em que há uma aparente divergência entre valores de uma mesma grandeza, medidas em condições distintas - poderia ser classificado de forma simplista na categoria de "montagem com equipamentos ruins". Mas o foco adequado deve ser as condições em que tais medições ocorreram, e este é um aspecto que merece ser ressaltado.

Muito embora a explicação para a diminuição da força de atrito estático durante o rolamento possa ser dada pela diminuição da componente normal da força de contato em alguns trechos, devido às ondulações da pista e ainda devido às possíveis excentricidade e "ovalização" da esfera, os autores gostariam de assumir o risco de propor um outro modelo. Neste modelo, a força normal média continuaria sendo dada por $m g \cos \theta$, em todo o movimento na pista e o resultado obtido em (13) seria o coeficiente de atrito estático "efetivo" entre as superfícies envolvidas. Diferentemente do coeficiente de atrito estático medido para o sistema isolado, este coeficiente de atrito estático "efetivo" caracterizaria não só o par de superfícies, em si, mas também as ondulações da pista, bem como a possível excentricidade da esfera, elementos que, no movimento, produzem a perturbação. Esta é uma maneira diferente de se abordar o problema, da qual se pode tirar muito proveito pedagógico, não só por envolver uma aparente discordância entre dois resultados obtidos para uma mesma grandeza, mas também por se tratar de um problema envolvendo uma situação real que normalmente não está presente em livros-texto de Física básica.

\section{Agradecimentos}

Gostaríamos de agradecer aos professores Dr. Antônio Almeida e Dr. Carlos Araújo, ambos do Departamento de Engenharia Mecânica de nossa universidade, pela leitura crítica de uma versão preliminar deste artigo.

\section{Referências}

[1] Caldas, H. e Saltiel, E., Rev. Bras. Ens. Fís. 21(3), 359 (1999).

[2] Caldas, H. e Saltiel, E., Rev. Bras. Ens. Fís. 21(4), 542 (1999).

[3] Praticamente todos os livros didáticos de Física para cursos superiores abordam, em um dos capítulos dedicados a Mecânica, o tema "força de atrito estático" em corpos que rolam sem deslizar.

[4] Mallinckrodt, A. J., Phys. Teach. 37, 463 (1999).

[5] Sherfinski, J., Phys. Teach. 39, 150 (2001).

[6] Takahashi, L., Phys. Teach. 40, 374 (2002).

[7] Brito, V. P. e Gomes, M. A. F., Rev. Bras. Ens. Fís. 18(1), 20 (1996).

[8] van den Berg, W. H., Phys. Teach. 38, 506 (2000).

[9] Johnson, G. O., Phys. Teach. 39, 154 (2001).

[10] Reichert, J. F., Phys. Teach. 39, 29 (2001).

[11] Mungan, C., E., Phys. Teach. 39, 481 (2001).

[12] Mossmann,V. L. F., Catelli, K. B. M. F., Libardi, H. e Damo, I. S., Rev. Bras. Ens. Fís. 24(2), 146 (2002).

[13] KEM - Kit para Experiências de Mecânica, info online, http://www.extensao.hpg.com.br/kits/kits.html

[14] Beer, F. P. e Johnston, E. R., Mecânica Vetorial para Engenheiros ESTÁTICA, Editora McGraw-Hill Ltda, São Paulo, $5^{a}$ Edição, (1990) pág. 574.

[15] Silva, Wilton P. e Silva, Cleide M. D. P. S., Mecânica Experimental para Físicos e Engenheiros, Ed. Universitária, João Pessoa (2000), pág. 54.

[16] LAB Fit Curve Fitting Software, online, download disponível em http://www.angelfire.com/rnb/labfit

[17] IrfanView, online, download disponível em http://www.Irfanview.com

[18] Bevington, P. R., e Robinson, D. K., Data Reduction and Error Analysis for the Physical Sciences, WCB/McGraw-Hill, Boston, Second Edition, (1992), Cap. 11.

[19] Silva, W. P. e Silva, C. M. D. P. S., Tratamento de Dados Experimentais UFPB Editora Universitária, João Pessoa, PB, $2^{a}$ Edição, (1998), seção 6.9.

[20] Tipler, P. A., Física, Editora Guanabara Dois S. A. Rio de Janeiro, RJ, Vol. 1, $1^{a}$ Edição, (1978), pág. 329.

[21] Vuolo, J. H., Fundamentos da Teoria dos Erros, Ed. Edgard Blücher Ltda São Paulo, SP, $1^{a}$ Edição, (1992), Cap. 8. 